\title{
Convolutional Neural Network Architecture for Plant Seedling Classification
}

\author{
Heba A. Elnemr \\ Computers and Systems Department \\ Electronics Research Institute \\ Cairo, Egypt
}

\begin{abstract}
Weed control is a challenging problem that may face crops productivity. Weeds are perceived as an important problem because they conduce to reduce crop yields due to the expanding competition for nutrients, water, and sunlight besides they serve as hosts for diseases and pests. Thus, it is crucial to identify weeds in early growth in order to avoid their side effects on crops growth. Previous conventional machine learning technologies exploited for discriminating crops and weeding species faced challenges of effectiveness and reliability of weed detection at preliminary stages of growth. This work proposes the application of deep learning technique for plant seedling classification. A new Convolutional Neural Networks (CNN) architecture is designed to classify plant seedlings at their early growth stages. The presented technique is appraised using plant seedlings dataset. Average accuracy, precision, recall, and F1score are utilized as evaluation metrics. The results reveal the capability of the proposed technique in discriminating among 12 species ( 3 crops and 9 weeds). The system achieved $94.38 \%$ average classification accuracy. The proposed system is compared with existing plant seedling systems. The results demonstrate that the proposed method outperforms the existing methods.
\end{abstract}

Keywords-Deep learning; convolutional neural network; plant seedling classification; weed control

\section{INTRODUCTION}

Plants remain an important and essential source of food and oxygen for nearly all living organisms on earth. Agriculture is prevailing in some continents like Africa, therefore appropriate automation of the farming procedure would assist in optimizing the crop yield and ensuring the perpetual productivity and sustainability. In accordance with [1], there is a sturdy bond between raised productivity and economic growth. Thus, the application of smart farming techniques in the agricultural sector can empower the development of the economy in many countries. Seedlings quality assessing proved to be a powerful means of prophesying the growth performance [2] and, hence, optimizing the plant production. Seedling classification is the first step to fulfill the seedling quality evaluation.

Furthermore, the invasion of weeds on farmlands leads to decline in the crop yield. Generally, weeds have no valuable beneficial, regarding nutrition, food or medication. However, they grow very quickly as well as they intrusively compete with original crops for space and nutrients [3]. Weeds identification is not an easy process due to the hazy boundaries of the crops, together with the diverse sandy and rocky backgrounds. Thus, there is a need to develop an efficient technique to accurately and certainly detect weeds from beneficial plants.

In order to improve agronomic production and crop quality, farmers should follow precision agriculture. Precision agriculture is a farm management approach that utilizes information technology and artificial intelligence to guarantee profit maximization, crop yield optimization, and environment preservation. One of the fundamental challenges that face precision agriculture is weed control. Weed control must be achieved earlier as possible after crop germination before weeds begin to compete with crops for nutrition and cause adverse effects. Thus, optimal weed treatment is recommended in the seedling stage. Nevertheless, in this phase, the discrimination between crops and weeds has some limitations; a) inadequate image resolution for distinguishing between exposed soil, crop seedlings and weeds, b) resemblance of spectra and appearances between weeds and useful crops in the early stages, and c) overlapping of the soil background reflectance with the detection process.

The application of machine learning techniques for automatic plant seedling classification has become a significant and promising field of research towards improving agriculture outcomes. Deep learning is a specific type of machine learning that has gained substantial interest in various disciplines. The Convolutional Neural Network (CNN) is a deep neural network architecture that is generally used to analyze visual images. Latterly, CNNs have achieved a significant breakthrough in computer vision fields. Additionally, the CNNs proved to have high ability to obtain the efficient features needed for image classification process [4]-[6].

Recently, CNNs have been broadly implemented in the agriculture domain for plant species identification [7]-[8], weed detection [9], and plant disease recognition [10].

In traditional image classification algorithms, handcrafted features are firstly extracted, then a feature selection process is achieved, and finally, a suitable classifier is chosen. However, $\mathrm{CNN}$ is proficient in learning various features from images, it covers global and local features, and it uses these features for efficient classification. CNN showed superior performance compared to other image processing techniques. Therefore, in this article, the enforcement of the CNN approach for plant seedling classification is investigated. The proposed system proceeds in four phases; preprocessing, constructing the network model architecture, training the network model and 
defining its parameters, and finally testing the designed network model. Despite the complexity of the acquired seedlings scenes due to illumination variations, resemblances between weeds and crops at premature stages of growth, and soil texture intricacies, the $\mathrm{CNN}$ succeeded to achieve high classification performance. Hence, this work aims specifically to develop a framework for crop-weed discrimination system that applies the CNN to classify 12 crops and weeds plant species and compare the proposed seedling classification system with other state-of-the-art techniques.

This article is structured as follows: Section 2 presents the related work. Section 3 details the CNN architecture devoted to developing the proposed deep plant seedling classification system. Section 4 describes and discusses the experimental results. Finally, Section 5 debates the conclusion and future work.

\section{RELATED WORK}

Seedlings classification is a discipline that has got a substantial prominence in precision agriculture, since it permits for distant observation to the fields, providing a foundation for more efficacious weed control. Fine-grained weed control considerably depends on the accuracy of the classification process, so as the crops would not be damaged when treating the weeds. Accordingly, misclassification will possess a direct impact on crop yield.

In literature, classification of crop and weed species may be developed through two strategies. The first strategy is based on segmenting images into green and soil regions and extracts features from green patches and finally uses classification techniques to obtain the specified classes. The second strategy, on the other hand, relies on implementing deep learning techniques for plant seedling classification.

The work of [11] presented a method for classifying plant seedlings. This method aimed to improve the classification performance by consolidating the classification of the whole plants and the individual leaves. Thus, leaves are first separated from the plants then features are extracted from both the whole plants and the segmented leaves. The classification process is performed for the leaves and plants, and finally, Bayes belief integration is used to fuse the classification results. Bakhshipour and Jafari [12] applied two significant pattern recognition approaches; artificial neural networks (ANN) and support vector machine (SVM), to separate the weeds from the sugar beet plants using shape features. The shape features comprise Fourier descriptors and moment invariant features. Four species of prevalent weeds in the sugar beet fields were examined. The results indicate that SVM slightly outperforms the ANN. In [13] the authors developed a system vision technique relied on video processing as well as a hybrid ANN and ant colony algorithm classifier for assorting potato plant and three weed species. Texture features, obtained from the gray level co-occurrence matrix (GLCM) and the histogram, moment invariants, color features, and shape features are extracted. Then, the Gamma test is used to select the significant features.

Furthermore, spectral reflectance measurements are used for discriminating between crops and weeds [14] and [15]. In
[14] an SVM along with spectral reflectance measurements are combined for developing a corn/silverbeet (as crop-weed) differentiation system. The intensities of the reflectance of laser beams off soil and vegetation at three wavelengths are gathered by a weed sensor. These reflectance measurements are used to compute the Normalized Difference Vegetation Indices (NDVIs). Two experiments are performed; in the first one, the obtained NDVI values are fed to an SVM to achieve the classification process, while in the second one, the raw reflected intensities are provided to the SVM for crop-weed discrimination. Strothmann et al. [15] proposed a crop-weed discrimination system based on in-field-labeling. A multiwavelength laser line profile (MWLP) approach is used to scan plants and obtain spectral reflection intensities, scattering information at several wavelengths and 3D data. The spectral features are applied for separating soil and biomass, while the 3D surface features are exploited for discriminating crops and weeds.

The study of [16] investigates the classification of maize, weeds, and soil by training $\mathrm{CNN}$ to make a pixel-wise classification. The generated $\mathrm{CNN}$ is based on a modified architecture of the VGG16 at which the output layer is a convolutional layer instead of a fully connected layer. Eventually, semantic segmented images are obtained. Zhang et al. [9] proposed a system for identifying broad-leaf weeds in the pasture. Traditional machine learning techniques and deep learning approaches are investigated and compared. The results reveal that deep learning technique using $\mathrm{CNN}$ achieved high accuracy and robustness in detecting weeds in real-world pasture environments. The work [17] submitted an approach to classify the species of weeds and crops by employing CNN technique. The developed CNN is based on a hybrid network of AlexNet and VGGNET. The normalization notion is stimulated from AlexNet; while the filters' depth is selected based on VGGNET. Furthermore, incremental learning to learn new plant species is applied in this work.

\section{PRoposed CNN ARCHITECTURE}

In this work, $\mathrm{CNN}$ is adopted for plant seedling classification to automatically discriminate between weed species and crops at early growth stages. The proposed CNN consists of an input layer, hidden layers, and an output layer. The original seedling images are all equally resized to $128 \times 128$ pixels (this has been specified empirically such that to get satisfactory performance with acceptable processing speed) and fed to the input layer. The hidden layers consist of 5 stages of learning layers, as illustrated in Fig. 1. The utilized filters are all of kernel size $3 \times 3$ with a number of filters $32,64,128$, 256 and 1024 for each convolutional layer within each stage, respectively.

The entire convolutional layers are associated with Rectified Linear Units (ReLU) layers, which apply the function $f(x)=\max (0, x)$ to the whole values of the input image. Thus, the negative input elements are set to 0 . This decreases the training time and provides nonlinear rectifications, which escalates the nonlinear characteristics of the model and the whole network without impacting the receptive values of the convolutional layer [18]. 


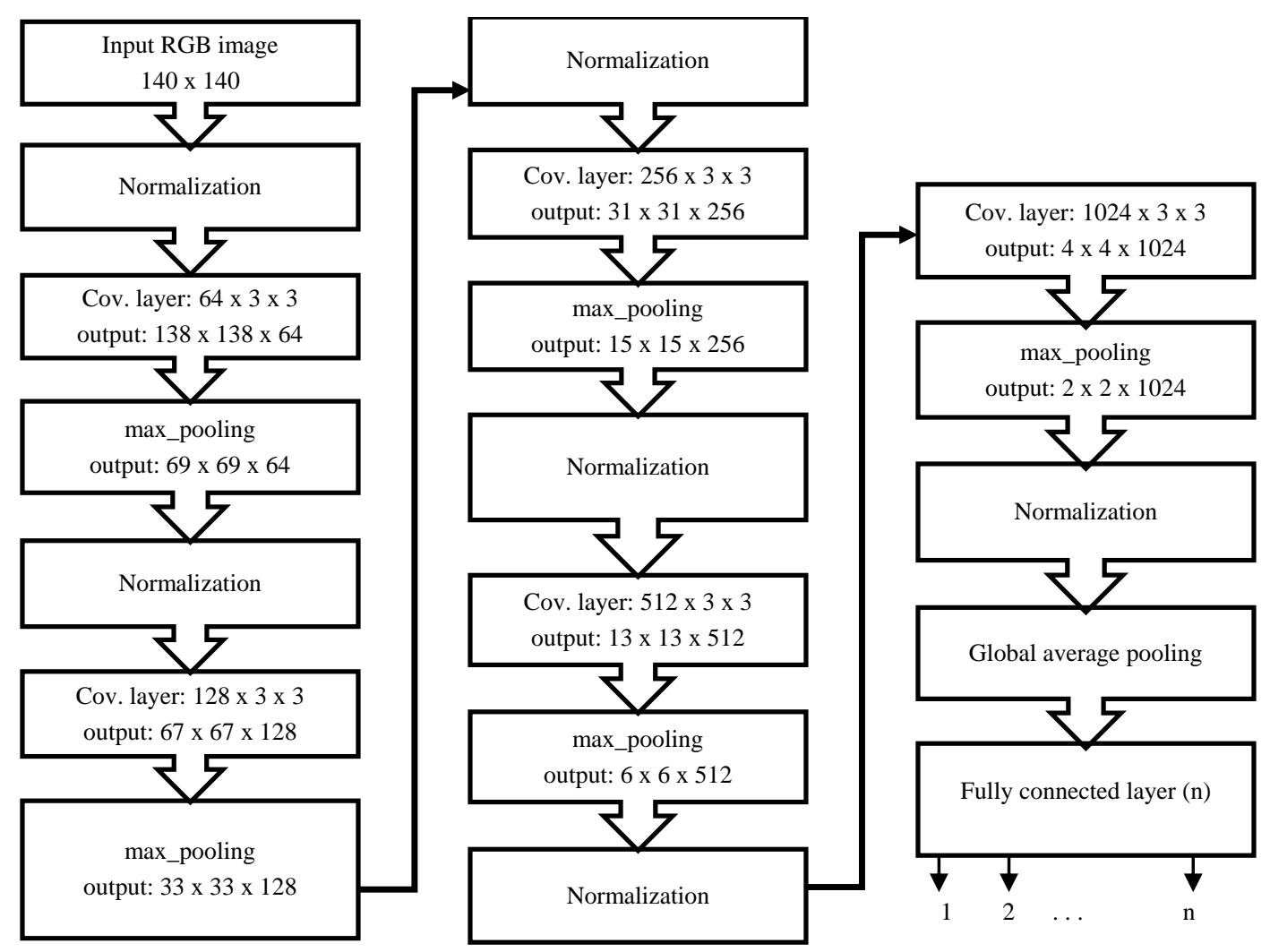

Fig. 1. The Proposed Deep CNN Architecture for Seedling Classification.

Each convolutional layer is followed by a pooling layer and a batch normalization layer. The pooling layer is utilized for reducing the output size of the layer before it, and hence, decreasing the computation complexity in the subsequent layers. A max-pooling procedure with a pool size of $2 \times 2$ is applied.

In deep $\mathrm{CNN}$, small variations may augment as they pass through layers, which lead to change the distribution within each layer. This is called Internal Covariate Shift problem. Therefore, batch normalization is utilized to normalize each hidden layer inputs to stabilize their distribution and hence solve the Internal Covariate Shift problem. Furthermore, the batch normalization layer helps to faster the learning procedure [19].

Generally, the convolutional layers are used for feature extraction and the fully connected layers are used for classification tasks. Thus, the lower part of the CNN includes convolutional layers while the higher part comprises some fully connected layers. The fully connected layers have a large number of parameters which needs a high computational power and produces overfitting. On the other hand, the global average layer procedure computes the mean of each feature map and delivers it to the next layer. Hence, it does not need any parameter which minimizes overfitting [18]. Our proposed $\mathrm{CNN}$ architecture employs the global average pooling layer before the fully connected layers in order to reduce the utilized parameters and avoid overfitting.

In the output layer, the global average pooling layer is used to directly feed the obtained feature maps into the feature vectors. Finally, a fully connected layer, which comprises $n$ (signifying the number of classes) nodes, along with softmax is realized to compute the probability of each predicted class.

The size of the output of each layer is declared in Fig. 1; after the normalization process, no change occurs in the output size.

\section{EXPERIMENTAL RESULTS}

\section{A. Dataset}

The utilized dataset, delivered by the signal processing group of the Aarhus University, in collaboration by Southern Denmark University, comprises 5539 images of roughly 960 unique plants categorized into 12 species ( 3 crops and 9 weeds) captured at early growth stages. It includes annotated RGB images with an approximate physical resolution of 10 pixels per $\mathrm{mm}$.

Particularly, this dataset is adopted for researches that investigate plant species identification at their early germination stage. Thus, farmers (or robots for automatic weeding control) may be able to handle weeding before the weeds commence to compete with crops for nutrition. Additionally, the image segmentation process at this early stage is easier since the leaves have less overlapping in this stage [20]. The exploited dataset is detailed in Table I.

\section{B. Evaluation Metrics}

The proposed system performance is evaluated using the average accuracy, average precision, average recall, and average F1-score as follows. 
TABLE. I. Plant SEedlings Dataset Details

\begin{tabular}{|c|c|c|c|c|}
\hline Class & Species & $\begin{array}{l}\text { Training } \\
\text { set images }\end{array}$ & $\begin{array}{l}\text { Test set } \\
\text { images }\end{array}$ & $\begin{array}{l}\text { Total } \\
\text { images }\end{array}$ \\
\hline 1 & $\begin{array}{l}\text { Black grass (Alopecurus } \\
\text { myosuroides) }\end{array}$ & 263 & 46 & 309 \\
\hline 2 & $\begin{array}{l}\text { Charlock (Sinapis } \\
\text { arvensis) }\end{array}$ & 390 & 62 & 452 \\
\hline 3 & $\begin{array}{l}\text { Cleavers (Galium } \\
\text { aparine) }\end{array}$ & 287 & 48 & 335 \\
\hline 4 & $\begin{array}{l}\text { Chickweed (Stellaria } \\
\text { media) }\end{array}$ & 611 & 102 & 713 \\
\hline 5 & $\begin{array}{l}\text { Common wheat } \\
\text { (Tricicum aestivum) }\end{array}$ & 221 & 32 & 253 \\
\hline 6 & $\begin{array}{l}\text { Fat hen (Chenopodium } \\
\text { 0album) }\end{array}$ & 475 & 63 & 538 \\
\hline 7 & $\begin{array}{l}\text { Loose silky-bent (Apera } \\
\text { spica-venti) }\end{array}$ & 648 & 114 & 762 \\
\hline 8 & Maize (Zea mays) & 221 & 36 & 257 \\
\hline 9 & $\begin{array}{l}\text { Scentless mayweed } \\
\text { (Tripleurospermum } \\
\text { perforatum) }\end{array}$ & 516 & 91 & 607 \\
\hline 10 & $\begin{array}{l}\text { Shepherd's purse } \\
\text { (Capsella bursa- } \\
\text { pastoris) }\end{array}$ & 231 & 43 & 274 \\
\hline 11 & $\begin{array}{l}\text { Small-flowered } \\
\text { Cranesbill (Geranium } \\
\text { pusillum) }\end{array}$ & 490 & 86 & 576 \\
\hline 12 & $\begin{array}{l}\text { Sugar beet (Beta } \\
\text { vugaris) }\end{array}$ & 385 & 78 & 463 \\
\hline Total & & 4738 & 801 & 5539 \\
\hline
\end{tabular}

average accuracy $=$

$\frac{1}{\mathrm{n}} \sum_{i=1}^{n}\left(\frac{\text { total number of correct samples for } \mathrm{i}^{\text {th }} \text { class }}{\text { number of total samples of } \mathrm{i}^{\text {th }} \text { class }}\right)$

average precision $=$

$\frac{1}{\mathrm{n}} \sum_{i=1}^{n}\left(\frac{\text { true positives }}{\text { true positives+false positives }}\right)_{\text {for } \mathrm{i}^{\text {th }}}$

average recall $=$

$\frac{1}{\mathrm{n}} \sum_{i=1}^{n}\left(\frac{\text { true positives }}{\text { true positives }+ \text { false negatives }}\right)_{\text {for } \mathrm{i}^{\text {th }}}$

average $\mathrm{F} 1-$ score $=$

$\frac{1}{\mathrm{n}} \sum_{i=1}^{n} 2 \times\left(\frac{\text { precision } \times \text { recall }}{\text { precision }+ \text { recall }}\right)_{\text {for } \mathrm{i}^{\text {th }}}$

\section{System Evaluation}

This section presents the evaluation strategy conducted through this work. The evaluation procedure is performed in two phases: training and testing. Thus, the seedling dataset is split into two separate sets; training and testing sets. In the training phase, 10-fold cross-validation is performed by randomly choosing $10 \%$ of the training set to represent the validation set, and this process is repeated for 10 successive rounds. In the training phase, the training images are used to fit the proposed $\mathrm{CNN}$ model and tune its hyperparameters while the validation images provide an unbiased appraisal of the model fitted on the training images during tuning its hyperparameters. On the other hand, in the test phase, the test images are utilized to afford an unbiased assessment of the final model that is fitted on the training dataset.

Extensive experimentations are achieved to evaluate the proposed method by comparing it with existing methods. These experiments are conducted by considering a different number of species. The first experiment involves 7 species (cleavers, chickweed, wheat, maize, scentless mayweed, Shepherd's purse and, sugar beet), the training set comprises 2472 images and the test set includes 430 images. The second experiment encompasses 8 species (charlock, cleavers, chickweed, fat hen, maize, scentless mayweed, Shepherd's purse, and sugar beet), the training phase contains (3116) images, whereas the testing phase holds (523) images.

The third experiment comprises 10 species (black grass, charlock, cleavers, chickweed, fat hen, loose silky-bent, maize, scentless mayweed, Shepherd's purse, and sugar beet), the training phase contains (4027) images, whereas the testing phase holds (683) images.

Finally, in the fourth experiment 12 species are considered and are divided into (4738) train images and (801) test images.

The proposed CNN is randomly initialized, and then it is trained for performing the classification process and indicated a convolutional model. The weights of the CNN are updated utilizing the training set. The final weights are selected using the validation set. For each iteration, the training and validation errors are computed, and the weights that achieve the minimum validation error are chosen.

Intel (R) Core (TM) i7-4702MQ CPU @ 2.20GHZ (8 GB RAM) processor is utilized for implementation. Python (Keras library) installed in Anaconda on the operating system Windows 10 is employed as a software tool for application.

\section{Results}

Table II presents the average validation performance of the proposed seedling classification for $7,8,10$ and 12 species. It can be noticed from Table II that the validation accuracy reaches approximately $99 \%$ for all tested number of species, the validation recall, precision, and F1-score are roughly $98 \%$ for 7 and 8 species while the validation recall reaches approximately $92 \%$ and $93 \%$ for the 10 and 12 species, respectively. In addition, the validation precision attains nearly $94 \%$ and $95 \%$ for 10 and 12 species, respectively, whereas the F1-score is about $93 \%$ for both 10 and 12 species.

On the other hand, Table III illustrates the average test performance. The results reveal that the average test accuracy, recall, precision, and F1-scale are approximately $99 \%$ for 7 species. For 8 species, the average test accuracy and recall are nearly $98 \%$ while the precision and F1-score are almost $99 \%$. Furthermore, when using 10 species, the average test accuracy, recall, precision and F1-scale reach roughly 95\%, 93\%, 97 and 94, respectively. Finally, as testing the 12 species, the average test accuracy and F1-score has attained approximately 94\%, while the average test recall and precision captured nearly $93 \%$ and $95 \%$, respectively. 
TABLE. II. The Average VAlidation PeRformance of the Proposed SYSTEM FOR 7, 8, 10 AND 12 SPECIES

\begin{tabular}{|l|l|l|l|l|l|}
\hline $\begin{array}{l}\text { Number of } \\
\text { plant species }\end{array}$ & $\begin{array}{l}\text { Accuracy } \\
(\boldsymbol{\%})\end{array}$ & $\begin{array}{l}\text { Recall } \\
(\boldsymbol{\%})\end{array}$ & $\begin{array}{l}\text { Precision } \\
(\boldsymbol{\%})\end{array}$ & $\begin{array}{l}\text { F1-score } \\
(\boldsymbol{\%})\end{array}$ & Loss \\
\hline 7 species & 98.63 & 97.9 & 98.2 & 97.79 & 0.0701 \\
\hline 8 species & 98.91 & 97.56 & 97.68 & 97.58 & 0.051 \\
\hline 10 species & 98.76 & 92.24 & 94.02 & 92.73 & 0.0489 \\
\hline 12 species & 99.01 & 92.64 & 94.86 & 92.93 & 0.0565 \\
\hline
\end{tabular}

TABLE. III. The Average Testing Performance of the Proposed SYSTEM FOR 7, 8, 10 AND 12 SPECIES

\begin{tabular}{|l|l|l|l|l|}
\hline $\begin{array}{l}\text { Number of } \\
\text { plant species }\end{array}$ & $\begin{array}{l}\text { Accuracy } \\
(\boldsymbol{\%})\end{array}$ & Recall $(\boldsymbol{\%})$ & $\begin{array}{l}\text { Precision } \\
(\boldsymbol{\%})\end{array}$ & $\begin{array}{l}\text { F1-score } \\
(\boldsymbol{\%})\end{array}$ \\
\hline 7 species & 98.61 & 98.63 & 98.92 & 98.77 \\
\hline 8 species & 98.28 & 98.38 & 98.59 & 98.47 \\
\hline 10 species & 94.88 & 93.22 & 96.47 & 94 \\
\hline 12 species & 94.38 & 93.1 & 94.83 & 93.57 \\
\hline
\end{tabular}

Moreover, the confusion matrices of the proposed seedling classification method for 7, 8, 10, 12 species are displayed in Table IV, Table V, Table VI, and Table VII, respectively.

TABLE. IV. THE CONFUSION MATRIX FOR 7 SPECIES (1. CLEAVERS, 2. ChickWeEd, 3. WheAt, 4. MAize, 5. SCEnTLESS MAYWEed, 6. SHEPHERD'S PURSE AND 7. SUGAR BEET)

\begin{tabular}{|c|c|c|c|c|c|c|c|c|}
\hline & & \multicolumn{7}{|c|}{ Predicted classes } \\
\hline & & 1 & 2 & 3 & 4 & 5 & 6 & 7 \\
\hline \multirow{7}{*}{ 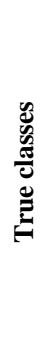 } & 1 & 48 & 0 & 0 & 0 & 0 & 0 & 0 \\
\hline & 2 & 0 & 100 & 0 & 0 & 2 & 0 & 0 \\
\hline & 3 & 1 & 0 & 31 & 0 & 0 & 0 & 0 \\
\hline & 4 & 0 & 0 & 0 & 36 & 0 & 0 & 0 \\
\hline & 5 & 0 & 1 & 0 & 0 & 89 & 0 & 1 \\
\hline & 6 & 0 & 0 & 0 & 0 & 1 & 42 & 0 \\
\hline & 7 & 0 & 0 & 0 & 0 & 0 & 0 & 78 \\
\hline
\end{tabular}

TABLE. V. THE CONFUSION MATRIX FOR THE 8 SPECIES (1. CHARLOCK, 2. Cleavers, 3. ChickweEd, 4. Fat Hen, 5. Maize, 6. SCEnTless MAYWEED, 7. SHEPHERD's PURSE AND 8. SUGAR BEET)

\begin{tabular}{|c|c|c|c|c|c|c|c|c|c|}
\hline & & \multicolumn{8}{|c|}{ Predicted classes } \\
\hline & & 1 & 2 & 3 & 4 & 5 & 6 & 7 & 8 \\
\hline \multirow{8}{*}{ 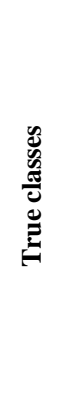 } & 1 & 60 & 1 & 0 & 0 & 0 & 0 & 1 & 0 \\
\hline & 2 & 0 & 48 & 0 & 0 & 0 & 0 & 0 & 0 \\
\hline & 3 & 0 & 0 & 101 & 0 & 0 & 1 & 0 & 0 \\
\hline & 4 & 0 & 0 & 2 & 61 & 0 & 0 & 0 & 0 \\
\hline & 5 & 0 & 0 & 0 & 0 & 36 & 0 & 0 & 0 \\
\hline & 6 & 0 & 0 & 3 & 0 & 0 & 88 & 0 & 0 \\
\hline & 7 & 0 & 0 & 0 & 0 & 0 & 1 & 42 & 0 \\
\hline & 8 & 0 & 0 & 0 & 0 & 0 & 0 & 0 & 78 \\
\hline
\end{tabular}

TABLE. VI. THE CONFUSION MATRIX FOR THE 10 SPECIES (1. BLACK Grass, 2. Charlock, 3. Cleavers, 4. ChickWeEd, 5. Fat Hen, 6. LoOSE SiLKy-Bent, 7. MAIZE, 8. SCENTLESS MAYwEed, 9. SHEPHERD's PuRSE AND 10. SUGAR BEET)

\begin{tabular}{|c|c|c|c|c|c|c|c|c|c|c|c|}
\hline & & \multicolumn{10}{|c|}{ Predicted classes } \\
\hline & & 1 & 2 & 3 & 4 & 5 & 6 & 7 & 8 & 9 & 10 \\
\hline \multirow{10}{*}{ 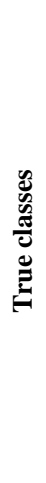 } & 1 & 21 & 0 & 0 & 0 & 0 & 25 & 0 & 0 & 0 & 0 \\
\hline & 2 & 0 & 62 & 0 & 0 & 0 & 0 & 0 & 0 & 0 & 0 \\
\hline & 3 & 0 & 0 & 47 & 0 & 0 & 0 & 0 & 0 & 0 & 1 \\
\hline & 4 & 0 & 0 & 0 & 100 & 0 & 0 & 0 & 2 & 0 & 0 \\
\hline & 5 & 0 & 0 & 0 & 2 & 61 & 0 & 0 & 0 & 0 & 0 \\
\hline & 6 & 2 & 0 & 0 & 0 & 0 & 112 & 0 & 0 & 0 & 0 \\
\hline & 7 & 0 & 0 & 0 & 0 & 0 & 0 & 36 & 0 & 0 & 0 \\
\hline & 8 & 0 & 0 & 0 & 2 & 0 & 0 & 0 & 89 & 0 & 0 \\
\hline & 9 & 0 & 0 & 0 & 0 & 0 & 0 & 0 & 1 & 42 & 0 \\
\hline & 10 & 0 & 0 & 0 & 0 & 0 & 0 & 0 & 0 & 0 & 78 \\
\hline
\end{tabular}

TABle. ViI. The Confusion Matrix for the 12 Species (1. Black Grass, 2. Charlock, 3. Cleavers, 4. Chickweed, 5. Wheat, 6. Fat Hen, 7. Loose Silky-Bent, 8. Maize, 9. Scentless Mayweed, 10. ShePherd’s Purse, 11. SMall-Flowered Cranesbill and 12. Sugar BeET)

\begin{tabular}{|c|c|c|c|c|c|c|c|c|c|c|c|c|c|}
\hline & & \multicolumn{12}{|c|}{ Predicted classes } \\
\hline & & 1 & 2 & 3 & 4 & 5 & 6 & 7 & 8 & 9 & 10 & 11 & 12 \\
\hline \multirow{12}{*}{ 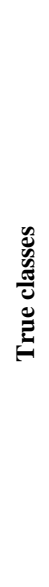 } & 1 & 20 & 0 & 0 & 0 & 1 & 0 & 25 & 0 & 0 & 0 & 0 & 0 \\
\hline & 2 & 0 & 60 & 0 & 0 & 0 & 0 & 0 & 0 & 0 & 0 & 2 & 0 \\
\hline & 3 & 0 & 0 & 48 & 0 & 0 & 0 & 0 & 0 & 0 & 0 & 0 & 0 \\
\hline & 4 & 0 & 0 & 0 & 100 & 0 & 0 & 0 & 0 & 2 & 0 & 0 & 0 \\
\hline & 5 & 1 & 0 & 0 & 0 & 31 & 0 & 0 & 0 & 0 & 0 & 0 & 0 \\
\hline & 6 & 0 & 0 & 0 & 2 & 1 & 60 & 0 & 0 & 0 & 0 & 0 & 0 \\
\hline & 7 & 6 & 0 & 0 & 0 & 0 & 0 & 108 & 0 & 0 & 0 & 0 & 0 \\
\hline & 8 & 0 & 0 & 0 & 0 & 0 & 0 & 0 & 36 & 0 & 0 & 0 & 0 \\
\hline & 9 & 0 & 0 & 0 & 3 & 0 & 0 & 0 & 0 & 88 & 0 & 0 & 0 \\
\hline & 10 & 0 & 0 & 0 & 1 & 0 & 0 & 0 & 0 & 1 & 41 & 0 & 0 \\
\hline & 11 & 0 & 0 & 0 & 0 & 0 & 0 & 0 & 0 & 0 & 0 & 86 & 0 \\
\hline & 12 & 0 & 0 & 0 & 0 & 0 & 0 & 0 & 0 & 0 & 0 & 0 & 78 \\
\hline
\end{tabular}




\section{E. Discussion}

In this section, an inclusive debate for the realized outcomes and comparison with state-of-art are exhibited.

As considering 7 species (3 crops and 4 weeds) and 8 species ( 2 crops and 6 weeds) experiments, the evaluation results depict that the proposed technique has effectively and efficiently classified the experimented species. The suggested system achieved about $99 \%$ average accuracy, recall, precision, and F1-score of for 7 species, and approximately $98 \%$ average accuracy and recall, as well as nearly $99 \%$ average precision and F1-score for 8 species.

On the other hand, for the 10 (2 crops and 8 weeds) and 12 ( 3 crops and 9 weeds) species, the performance evaluation is relatively less than that for 7 and 8 species. Despite the performance reduction, the classification evaluation still high and the empirical results manifest the potency and capability of the proposed system in discriminating among the various species. The submitted method, for 10 species, obtained average accuracy, recall, precision and F1-score of about $95 \%$, $93 \%, 97 \%$, and $94 \%$, respectively. Additionally, for 12 species, the system attained approximately 94\%, 93\%, 95\% and 94\% average accuracy, recall, precision, and F1-score, respectively. It is apparent from Table VI and Table VII that Black grass and Loose silky-bent majorly affect the results due to their high similarity and insignificant differences at early-stage growth, and they are hard to be distinguished even by human eyes. As for other classes, the proposed model proved to be reliable and effective.

To scrutinize the performance of the proposed technique, a comparison is performed with some state-of-the-art. The proposed method is compared with the existing methods [21], [11] and [17].

The average accuracy of the existing seedling approaches and the proposed work are quoted in Table VIII. It may be observed that the proposed deep seedling classification system outperforms significantly [21] and [11]. Furthermore, it can be noticed that, in [11], the average accuracy for Cleavers and Fat hen classes are $81.4 \%$ and $81.6 \%$, respectively, which are relatively weak. Yet, the proposed technique has developed it to $100 \%$ and $96.83 \%$, respectively. Moreover, both works [11] and [21] skipped pretty similar species like black grass and loose silk bent. However, the submitted seedling classification approach achieves an average test accuracy of $94.38 \%$ for the whole 12 species.

TABLE. VIII. COMPARISON OF THE PROPOSED METHOD AND EXISTING METHODS

\begin{tabular}{|l|l|l|}
\hline Method & Number of species & Accuracy \\
\hline$[21]$ & 7 & 95.8 \\
\hline$[17]$ & 7 & 98.21 \\
\hline Proposed method & 7 & $\mathbf{9 8 . 6 1}$ \\
\hline$[11]$ & 8 & 96.7 \\
\hline$[17]$ & 8 & 98.23 \\
\hline Proposed method & 8 & $\mathbf{9 8 . 2 8}$ \\
\hline$[17]$ & 12 & 93.64 \\
\hline Proposed method & 12 & $\mathbf{9 4 . 3 8}$ \\
\hline
\end{tabular}

Over and above, the proposed system performance slightly exceeds that of [17] for 7 and 8 species, and significantly outperforms it for 12 species. Furthermore, it involves 6 convolutional layers and 3 fully-connected layers, whereas our proposed CNN comprises 5 convolutional layers and one fully connected layer. Adding more layers extends the number of hyperparameters, ergo the complexity of the system. Thus, the submitted $\mathrm{CNN}$ architecture is much simpler and provide superior performance.

\section{CONCLUSION}

In this article, a $\mathrm{CNN}$ architecture is developed to discriminate between plant images of crop species and weed species at several early growth stages. The proposed CNN has achieved an enhancement in performance owing to the combination of the presence of the normalization layer, the global average pooling layer and the choice of the depth of the filters. The results revealed that the elaborated CNN has an encouraging performance towards building a weed control system which is a step to precision agriculture. The proposed CNN model achieved average accuracy, recall, precision, and F1-score of 94.38, 93.1, 94.83, and 93.57, respectively, for discriminating 12 plant seedling (3 crops and 9 weeds). Furthermore, its architecture is simpler than other existing CNN models utilized for plant seedling classification. Additionally, its performance is much better than other existing methods.

In the proposed scheme, images that comprise single plant species are classified, thus, for classifying images with many plant species, the segmentation stage may be added to the system.

In addition, the proposed technique may be expanded to incorporate new plant species. Besides, the proposed technique may be implemented as a part of an IoT system for weed control, which can help to directly apply herbicides on the weeds without harming crops.

\section{REFERENCES}

[1] The Role of International Institutions in Economic Development and Poverty Reduction in the Developing World, Food and agriculture Organization of the United Nations. Rome, 2018. URLhttp://www.fao.org/3/I9900EN/i9900en.pdf

[2] L.O.L.A. Silva, M.L. Koga, C.E. Cugnasca, and A.H.R. Costa, "Comparative assessment of feature selection and classification techniques for visual inspection of pot plant seedlings", Computers and Electronics in Agriculture vol. 97, pp. 47-55, 2013.

[3] D. Nkemelu, D. Omeiza, and N. Lubalo, "Deep convolutional neural network for plant seedlings classification", Computer Vision and Pattern Recognition, arXiv: 1811.08404, 2018.

[4] A. Dhomne, R. Kumar, and V. Bhan, "Gender recognition through face using deep learning”, Procedia Computer Science, vol. 132, pp. 2-10, 2018.

[5] M. Radovic, O. Adarkwa, and Q. Wang, "Object recognition in aerial images using convolutional neural networks", Journal of Imaging, vol. 3, no. 21, 2017.

[6] N. M. Zayed and H. A. Elnemr, "Intelligent Systems for Healthcare Management and Delivery", chapter 5, "Deep Learning and Medical Imaging”, pp. 101-147, 2019.

[7] P. Bonnet, H. Goëau, S. Hang, M. Lasseck, M. Šulc, V. Malécot, P. Jauzein, J. Melet, C. You, and A. Joly, "Plant identification: experts vs. machines in the era of deep learning: deep learning techniques challenge flora experts". In: A. Joly, S. Vrochidis, K. Karatzas, A. Karppinen, P. Bonnet (eds.). Multimedia Tools and Applications for Environmental \& 
Biodiversity Informatics. Multimedia Systems and Applications. Springer, Cham, Chapter 8, pp.131-149, 2018.

[8] Z. Qiu, J. Chen, Y. Zhao, S. Zhu, Y. He, and C. Zhang, "Variety identification of single rice seed using hyperspectral imaging combined with convolutional neural network", Applied Sciences, vol. 8, no. 2, 212, 2018.

[9] W. Zhang, M. F. Hansen, T. N. Volonakis, M. Smith, L. Smith, J. Wilson, G. Ralston, L. Broadbent, and G. Wright, "Broad-Leaf weed detection in pasture," 2018 IEEE 3rd International Conference on Image, Vision and Computing (ICIVC), Chongqing, pp. 101-105, 2018.

[10] K. P. Ferentinos, "Deep learning models for plant disease detection and diagnosis". Computers and Electronics in Agriculture, vol. 145, pp. 311318, 2018.

[11] M. Dyrmann, P. Christiansen, and H. S. Midtiby, "Estimation of plant species by classifying plants and leaves in combination", Journal of Field Robotics, vol. 35, pp. 202-212, June 2017.

[12] A. Bakhshipour, and A. Jafari, "Evaluation of support vector machine and artificial neural networks in weed detection using shape features", Computers and Electronics in Agriculture, vol. 145, pp. 153-160, 2018.

[13] S. Sabzla, Y. A. Gilandeha, and H. Javadikia, "Developing a Machine Vision System to Detect Weeds from Potato Plant", Journal of Agricultural Sciences, vol. 24, pp. 105-118, 2018.

[14] S. Akbarzadeh, A. Paap, S. Ahderom, B. Apopei, and K. Alameh, "Plant discrimination by Support Vector Machine classifier based on spectral reflectance", Computers and Electronics in Agriculture, vol. 148, pp. 250-258, 2018.
[15] W. Strothmann, A. Ruckelshausen, J. Hertzberg, C. Scholz, and F. Langsenkamp, "Plant classification with In-Field-Labeling for crop/weed discrimination using spectral features and 3D surface features from a multi-wavelength laser line profile System", Computers and Electronics in Agriculture, vol. 134, pp. 79-93, 2017.

[16] M. Dyrmann, A. K. Mortensen, H. S. Midtibya, and R. N. Jørgensen, "Pixel-wise classification of weeds and crop in images by using a Fully convolutional neural network", International Conference on Agricultural Engineering 2016 - Aarhus University, Aarhus, Denmark, June 26-29, 2016.

[17] T. R. Chavan, and A. V. Nandedkar, "AgroAVNET for crops and weeds classification: A step forward in automatic Farming", Computers and Electronics in Agriculture, vol. 154, pp. 361-372, 2018.

[18] A. Krizhevsky, I. Sutskever, and G. Hinton, "Imagenet classification with deep convolutional neural networks", Twenty-sixth Conference on Neural Information Processing Systems (NIPS), December 3-8, 2012.

[19] S. Ioffe, and C. Szegedy, "Batch normalization: Accelerating deep network training by reducing internal covariate shift", The 32nd International Conference on Machine Learning (ICML 2015), Lille, France, July $6-11,2015$.

[20] T. Giselsson, R. Jørgensen, P. Jensen, M. Dyrmann, and H. Midtiby, “A public image database for benchmark of plant seedling classification algorithms", Computer Vision and Pattern Recognition, arXiv:1711.05458, November, 2017.

[21] P. Christiansen, and M. Dyrmann, "Automated classification of seedlings using computer vision", Technical report, Aarhus University, Aarhus, 2014. 